\title{
Tournament-stable equilibria
}

\author{
Francesco De Sinopoli ${ }^{a}$, Claudia Meroni ${ }^{\mathrm{a}, *}$, Carlos Pimienta ${ }^{\mathrm{b}}$ \\ ${ }^{a}$ Department of Economics, University of Verona, Verona, Italy \\ b School of Economics, The University of New South Wales, Sydney, Australia
}

\section{A R T I C L E I N F O}

\section{Article history:}

Received 23 July 2019

Received in revised form 9 October 2019

Accepted 11 November 2019

Available online 18 November 2019

\section{Keywords:}

Tournaments

Asymmetric contests

Refinement criterion

\begin{abstract}
A B S T R A C T
Building on Arad and Rubinstein (2013), we introduce tournaments as simultaneous $n$-player games based on an $m$-player game $g$. A player meets each group of $m-1$ opponents $m$ ! times to play $g$ in alternating roles. The winner of the tournament is the player who attains the highest accumulated score. We explore the relationship between the equilibria of the tournament and the equilibria of the game $g$ and confirm that tournaments provide a refinement criterion. We compare it with standard refinements in the literature and show that it is satisfied by strict equilibria. We use our tournament model to study a selection of relevant economic applications, including risk-taking behavior.
\end{abstract}

(c) 2019 Elsevier B.V. All rights reserved.

\section{Introduction}

The two-player game

$$
\begin{array}{c|c|c}
\multicolumn{1}{c}{} & \multicolumn{1}{c}{C} & \multicolumn{1}{c}{D} \\
\cline { 2 - 3 } A & 6,5 & 4,3 \\
\cline { 2 - 3 } B & 4,0 & 3,1 \\
\cline { 2 - 3 } & &
\end{array}
$$

has the unique Nash equilibrium $(A, C)$. Suppose that two players play this game twice, simultaneously, and in alternating roles, with the objective of maximizing the sum of the payoffs they get in both matches. That is, they play the symmetric game

\begin{tabular}{c|c|c|c|c}
\multicolumn{1}{c}{$A C$} & \multicolumn{1}{c}{$A D$} & \multicolumn{1}{c}{$B C$} & \multicolumn{1}{c}{$B D$} \\
\cline { 2 - 5 }$A C$ & 11,11 & 9,9 & 6,9 & 4,7 \\
\cline { 2 - 5 }$A D$ & 9,9 & 7,7 & 7,8 & 5,6 \\
\cline { 2 - 5 }$B C$ & 9,6 & 8,7 & 4,4 & 3,5 \\
\cline { 2 - 5 }$B D$ & 7,4 & 6,5 & 5,3 & 4,4 \\
\cline { 2 - 5 } & & & &
\end{tabular}

If they both play $A C$ - i.e. $A$ when in the role of player 1 and $C$ when in the role of player 2 - they both get a payoff of 11 . None of them has an incentive to deviate, since any other choice leads to a lower payoff, and both players playing $A C$ is the unique equilibrium of such a game. ${ }^{1}$

Consider the same situation with the difference that the numbers that represented payoffs are now just scores, a prize is

\footnotetext{
A preliminary analysis of the case in which the base game is a two-player game is contained in the working paper "Double round-robin tournaments" (UNSW Business School Research Paper No. 2016 ECON 04).

* Corresponding author.

E-mail addresses: francesco.desinopoli@univr.it (F. De Sinopoli), claudia.meroni@univr.it (C. Meroni), c.pimienta@unsw.edu.au (C. Pimienta).

1 In fact, it is the only strategy that survives iterated deletion of strictly dominated strategies.
}

awarded to the player with the highest total score, and players want to maximize the probability of winning the prize. In the event of a tie, each player is the winner with probability $1 / 2$. In this case $(A C, A C)$ is no longer an equilibrium. ${ }^{2}$ Indeed, each player has an incentive to deviate, for instance, to $B C$. In doing so, the deviator loses two points but also lowers her opponent's payoff by five points, thus winning the prize with probability 1 . It is easy to see that the unique equilibrium of this game is $(B D, B D)$ and that both players win with probability $1 / 2 .^{3}$

We can think of the same competitive situation extended to any number $n$ of players, where everyone meets all her opponents twice to play the same two-player game in alternating roles and where the prize is awarded to the player with the highest accumulated score. We call such a competition "double roundrobin tournament". Furthermore, we can extend this competition model to any possible $m$-player base game, so that each player plays $g$ with every possible group of $m-1$ opponents $m$ ! times, each time in a different permutation of the $m$ roles. We call such a competition " $m$-tuple round-robin tournament", or simply "tournament".

\footnotetext{
2 Note that, with this notation, we list a strategy for each player that indicates first what she chooses when in the role of player 1 and then what she chooses when in the role of player 2 . For instance, the profile $(A C, B D)$ represents the case in which in one match $(A, D)$ is played and in the other $(B, C)$ is played.

3 Note that the original game is transformed into the following $4 \times 4$ constant sum game:
}

\begin{tabular}{c|c|c|c|c|}
\multicolumn{1}{c}{$A C$} & \multicolumn{1}{c}{$A D$} & \multicolumn{1}{c}{$B C$} & $B D$ \\
\cline { 3 - 5 }$A C$ & $1 / 2,1 / 2$ & $1 / 2,1 / 2$ & 0,1 & 0,1 \\
\cline { 2 - 5 }$A D$ & $1 / 2,1 / 2$ & $1 / 2,1 / 2$ & 0,1 & 0,1 \\
\cline { 2 - 5 }$B C$ & 1,0 & 1,0 & $1 / 2,1 / 2$ & 0,1 \\
\cline { 2 - 5 }$B D$ & 1,0 & 1,0 & 1,0 & $1 / 2,1 / 2$ \\
\cline { 2 - 5 } & & &
\end{tabular}


This construction is based on round-robin tournaments as introduced by Arad and Rubinstein (2013) (henceforth, AR13). An $n$-player round-robin tournament $R(g, n)$ is a simultaneous game in which every player is matched with each one of her $n-1$ opponents once to play the two-player symmetric game $g$. AR13 assume that each player uses the same action every time she meets a new opponent. In turn, we define an $m$-tuple round-robin tournament (or simply tournament) $T(g, n)$ as a simultaneous $n$-player game in which every player is matched with each group of $m-1$ opponents $m$ ! times and, in every match, a (possibly asymmetric) $m$-player game $g$ is played, where $m \leq n$. In the $m$ ! matches of the same group of players, they play $g$ in all the possible permutations of the $m$ roles. We assume that each player always uses the same action in all the matches that she plays in a given role, independently of the set of opponents of any given match and the roles in which they play. The winner of the tournament is the player with the highest accumulated score, which is given by the sum of the scores that she obtains in all the matches she plays. Players care only about maximizing the probability of being the highest scorer in the tournament and not about maximizing the total score. ${ }^{4}$

Like with the opening example, throughout our analysis we often consider the simplest case in which $g$ is a two-player game. To this end we also define a double round-robin tournament $D(g, n)$ as an $n$-player tournament based on a (possibly asymmetric) twoplayer game $g$. A player plays $g$ twice with each opponent, once in the role of the first player and once in the role of the second player of $g$. Using sports terminology, we say that a player plays once "at home" and once "away". Thus, each player always uses the same action in all the matches she plays at home and the same action in all the matches she plays away.

We follow AR13 and use the symmetric Nash equilibrium as solution concept, so that every player uses the same strategy that specifies which action to play in each of the $m$ roles. ${ }^{5}$ The vector of actions that a player will ultimately play in each role is the outcome of a randomization (mixed strategy) executed only once and before starting the interactions within the tournament. Consider again the initial example. Under the assumptions of our model, the tournament with three players has a symmetric equilibrium in which every player chooses $B$ when playing at home and $D$ when playing away. For $n \geq 4$, instead, the symmetric Nash equilibrium of the tournament prescribes players to choose $A$ at home and $C$ away, which are the Nash equilibrium strategies of the original two-player game. As with AR13, this is not a coincidence. As $n$ grows, deviating from the equilibrium strategies of the base game becomes less and less profitable. The potential loss inflicted to each of the other players becomes negligible compared to the score foregone by not playing a best response to the strategy used by every other player in the tournament. The consequence is that the limit as $n$ goes to infinity of a sequence of equilibria of $T(g, n)$ must be a Nash equilibrium of $g$.

However, the opposite is not true. There may be equilibria of the base game $g$ that are not limit points of equilibria of tournaments as the number of players grows to infinity. Thus,

\footnotetext{
4 In the example at the beginning we have seen that, when individuals want to maximize the sum of the payoffs in the two matches, the unique equilibrium prescribes the Nash equilibrium strategies of the base game. In general it is clear that if players' objective is to maximize the total accumulated score and players employ the same action in all the matches they play in a given role then every equilibrium of the base game is a symmetric equilibrium of the tournament and vice versa. As discussed at the end of the introduction, see Laffond et al. (2000) for an analysis of the case in which the base game is a symmetric two-player game.

5 As we will see in Section 3, the use of symmetric Nash equilibrium is necessary to properly relate equilibria of the tournament and equilibria of the game on which it is built. In fact, a strategy of the tournament identifies a strategy combination of the base game.
}

we study the conditions under which equilibria of $g$ are an appropriate approximation of equilibria of the tournament. In this way, we extend to any $m$-player (and possibly asymmetric) game $g$ the criterion that selects a subset of Nash equilibria as "a stable distribution of actions over a large population of individuals who are occasionally matched to play $g[\ldots]$ in environments where individuals are interested in maximizing the chances of being the best" (AR13, p.33). We call these equilibria "tournament-stable".

The standard literature on equilibrium refinements has provided several solution concepts that restrict the set of Nash equilibria in order to satisfy rationality requirements, e.g. existence, admissibility, iterated dominance, backward and forward induction (see van Damme, 1991 for a survey). We compare the criterion provided by tournaments with some of these solution concepts. We show that strict equilibria (i.e. Nash equilibria such that, for every player, every deviation implies a strict loss) are always tournament-stable. We then present two classic economic applications, the ultimatum game and the entry game, in which tournaments and prominent equilibrium refinements lead to different results. In the ultimatum game the set of tournamentstable equilibria appears to be too restrictive, as it does not contain the equilibrium in which the first player makes an unfair proposal and the second player accepts it, which is a Kohlberg and Mertens (1986) stable set as a singleton. ${ }^{6}$ However, in the entry game, the concept of tournament-stable equilibrium proves to be too weak, as it sustains all the dominated Nash equilibria in which the potential entrant stays out of the market because the incumbent is going to behave aggressively with some positive probability.

AR13 list different interpretations of their tournament model. We note that most of them can be extended to also motivate our tournaments, with the additional advantage of being able to study any asymmetric interaction. According to the straightforward interpretation, players can participate in a tournament where changing action from match to match is costly enough so that they have to use the same action in all the matches. Double round-robin tournaments, for instance, are common in several sports competitions, where each team plays against every other team home and away. In such competitions, the coach, players and tactics have to be decided before the season starts and matches are typically asymmetric since the home team has the home court advantage. According to the social interpretation, agents can be occasionally involved in asymmetric interactions and only care about being the best achiever. Often, they play alternating roles in such interactions. For instance, they are both buyers and sellers, give and make interviews, make or receive the first offer when bargaining, etc. Finally, individuals can be randomly matched to interact in a biological contest. This is typically asymmetric, since individuals differ in qualities such as size, age, fighting ability, etc., which affect their relative probability of winning an isolated contest as well as how much they have to gain from it. The asymmetry can also be arbitrary and arise from the fact that one of the contestants arrives at the resource first and becomes the owner. Behavior can be conditioned on such asymmetries and can be summarized by a strategy that is genetically determined such as "if you are the owner of the resource attack, if you are the intruder retreat" (Maynard Smith and Parker, 1976). Evolution selects only the highest achieving strategies and eliminates the rest.

As argued by AR13, the refinement criterion provided by tournaments may be more appropriate to such an evolutionary interpretation of equilibrium. Indeed, every Evolutionary Stable Strategy is robust to AR13's tournament model. The fact that evolution

\footnotetext{
6 Loosely speaking, a Kohlberg and Mertens (1986) stable set is a minimal subset of Nash equilibria that is robust against every possible strategy perturbation; that is, every close-by game that can be generated through pure-strategy perturbations has a Nash equilibrium close to the stable set.
} 
only selects the best performing strategies intuitively encourages risky behavior. Similarly to AR13, we also study whether tournaments favor risky strategies. We do this using a novel and very natural framework in which tournaments turn out to be an extremely useful refinement criterion. We consider a two-player game in which player 1 has to choose between a safe outside option or entering a constant sum game against player 2 , whose Nash equilibrium payoff to player 1 coincides with that of the outside option. We demonstrate that the tournament structure incentivizes engaging in risk-taking behavior as, in the unique tournament-stable equilibrium, player 1 chooses to play the constant sum game with probability 1 . We also show that this result is exactly driven by tournament incentives. Indeed we show that if players wanted to minimize the probability of being the lowest scorers then in the unique tournament-stable equilibrium player 1 would always choose the safe outside option.

The applications of tournaments that we present consist of games that have been traditionally studied by the experimental literature. In fact, AR13 (p.34) justify the tournament model also in the context of experimental design. To study behavior in the game $g$ one may propose an experiment consisting of a group of players who are re-matched after each interaction and incentivized by the promise of a prize to the highest scorer. An obvious caveat to this design is that it creates incentives that are different from those present in $g$, as evidenced by the fact already discussed that the set of equilibria of $g$ and of the tournament can be different. We emphasize that our model allows to extend such a scope to all the possible games studied in experiments, which are typically asymmetric.

Our work expands AR13's analysis to every possible game and to every possible equilibrium. In fact, not only can we study the equilibria of non-trivial extensive form games, but also the nonsymmetric equilibria of symmetric games, which in many cases are the more economically relevant equilibria. ${ }^{7}$ In particular, we extend AR13's analysis using a symmetrization of the game under study. ${ }^{8}$ We relate strategy combinations (and equilibria) of the base game with strategies (and symmetric equilibria) of such a construction by proving an analogue of Kuhn's theorem (Kuhn, 1953). This allows us to generalize AR13's Proposition 1, which establishes that every limit equilibrium of tournaments is an equilibrium of the base game. Moreover, we present an example which shows that there can be equilibria of a game that are not limit equilibria of tournaments built on it, replicating also AR13's Proposition 2. Then, we discuss tournament-stability as an equilibrium refinement with classical examples of extensive form games in which the information structure is crucial. To complete the comparison with AR13 we conclude analyzing some two-player symmetric games, on which we can build both our double round-robin tournaments and their round-robin tournaments. The results obtained in the two cases show how AR13's conclusions do not extend immediately to our setting.

7 Think for instance of the "car-driving game"

\begin{tabular}{l|c|c|}
\multicolumn{1}{c}{} & \multicolumn{1}{c}{$L$} & \multicolumn{1}{c}{$R$} \\
\cline { 2 - 3 }$L$ & 0,0 & 1,1 \\
\cline { 2 - 3 }$R$ & 1,1 & 0,0 \\
\cline { 2 - 3 }
\end{tabular}

where the only symmetric equilibrium is in mixed strategies, while the two pure strategy equilibria in which the two drivers choose different lanes are non-symmetric. Also, consider the "battle of the sexes", where again the unique symmetric equilibrium is in mixed strategies, but it is Pareto dominated by each of the two pure-strategy non-symmetric equilibria. It is unlikely that such a symmetric equilibrium emerges from the stage of pre-play communication and bargaining on which the theory of equilibrium is based and during which players coordinate on one of the equilibria (of course, no agreement concluded at that stage is enforceable).

8 In the initial example, it corresponds to the symmetric $4 \times 4$ game in which payoffs are the sum of the payoffs in the two matches.
An alternative tournament model is considered by Laffond et al. (2000). As in AR13 and in our model, each player chooses one action and employs it in all her interactions. However, a player's payoff is given by the sum of the payoffs that she gets in all the (symmetric) games she plays, so players do care about their absolute total score. AR13 already explain the differences between the tournament model and the classic model of contests (see, e.g., Green and Stokey 1983, Dixit 1987, Konrad 2009; among others), where players' utilities depend on the probability of winning, which is a function of players' efforts, and on the cost of their own effort. A particular contest model is that of the elimination tournament, which consists of several rounds in which individuals play pairwise matches (see, e.g., Rosen 1986, Konrad 2004, Groh et al. 2012). Differently from our model, the winner of a match advances to the next round of the tournament, while the loser is eliminated from the competition.

We formally describe the model in the next section. In Section 3, we analyze the interaction between any $m$ players in the tournament. We examine the relationship between equilibria of the tournament and equilibria of the game on which it is built in Section 4, and we compare the refinement criterion provided by tournaments with standard refinements in Section 5 . Section 6 explores risk-taking incentives in tournaments, while Section 7 extends the comparison between double round robins and AR13's round robins.

\section{The model}

An m-tuple round-robin tournament (or simply tournament) $T(g, n)$ is a simultaneous $n$-player game built on an $m$-player normal form game $g:=\left(M,\left(S_{i}\right)_{i \in M},\left(u_{i}\right)_{i \in M}\right)$. We often refer to $g$ as a match, and we identify each player of $g$ with a role. The set $M=\{1, \ldots, m\}$ is the set of roles in the match, where $m \leq n$. Every player plays $g$ against each possible group of $m-1$ other players and in all the possible permutations of the $m$ roles. In particular, when $m=2$, every player plays $g$ with each other player twice, once in the role of the first player of $g$ (which, using sports terminology we refer to as the player at home) and once in the role of the second player of $g$ (or away player). Thus, the total number of matches in the tournament is given by $\left(\begin{array}{l}n \\ m\end{array}\right) m !=\frac{n !}{(n-m) !}$, that is, the number of possible groups of $m$ players multiplied by the number of possible permutations of the $m$ roles.

For each $i \in M$, the finite set $S_{i}$ is the set of actions available to a player who undertakes role $i$. Every player is assumed to employ the same action $s_{i} \in S_{i}$ in all the matches she plays in role $i$. The function $u_{i}: \prod_{i \in M} S_{i} \rightarrow \mathbb{R}$ specifies the score that a player in role $i$ obtains for each action profile in the match. That is, when she plays $s_{i}$ and her opponents play according to $s_{-i}=\left(s_{1}, \ldots, s_{i-1}, s_{i+1}, \ldots, s_{m}\right)$ in a match, her score in that match is $u_{i}\left(s_{i}, s_{-i}\right)$. A player's total score is the sum of the scores that she gets in the $\frac{(n-1) !}{(n-m) !} m$ matches she participates in. ${ }^{9}$ The player with the highest total score wins the tournament. In the case of a tie, the winner is chosen randomly among the set of top-scoring players. Each player's objective is to maximize her probability of winning the tournament.

A pure strategy $s$ in the tournament is a mapping that specifies for each role $i$ which action $s_{i}$ to take in the matches played in role $i$. The set of pure strategies of each player is $S:=\prod_{i \in M} S_{i}$. That is, it is the set of pure strategy combinations of the match. A mixed strategy $\sigma$ is an element of $\Sigma:=\Delta(S)$, the set of probability distributions on $S$. Following AR13 we assume global randomization, that is, mixed strategies are executed only once and the player

\footnotetext{
9 The number of matches that each player plays is equal to the number possible groups of $m-1$ opponents, $\left(\begin{array}{c}n-1 \\ m-1\end{array}\right)$, multiplied by the number of times she plays with each of those possible groups, $m$ !.
} 
employs the actions $s_{1}, \ldots, s_{m}$ dictated by the resulting pure strategy in all the matches she plays in role $1, \ldots, m$ respectively.

Given the structure of the problem, the tournament is a symmetric game (even if the match $g$ is usually not) and we focus on symmetric Nash equilibria in which every player plays the same mixed strategy. Let $P\left(\sigma^{\prime}, \sigma\right)$ be the probability that a given player wins the tournament when she plays the mixed strategy $\sigma^{\prime}$ and her $n-1$ opponents play $\sigma$.

Definition 1. The mixed strategy $\sigma$ is an equilibrium of the tournament $T(g, n)$ if

$P(\sigma, \sigma) \geq P\left(\sigma^{\prime}, \sigma\right) \quad$ for all $\sigma^{\prime} \in \Sigma$.

The set $\Sigma$ is nonempty, compact, and convex, and the function $P\left(\sigma^{\prime}, \sigma\right)$ is linear in $\sigma^{\prime}$ and continuous in $\sigma$, making the associated best response correspondence convex-valued and upper semicontinuous. Thus, every tournament has an equilibrium in mixed strategies. Notice that, if $\sigma$ is an equilibrium of the tournament, every action in the support of $\sigma$ wins with probability $1 / n$ when all the other $n-1$ players play $\sigma$. In turn, every action that is not in the support of $\sigma$ wins the tournament with probability not greater than $1 / n$.

\section{The strategies}

A player in the tournament interacts $m$ ! times with each group of opponents, playing the match $g$ against them in all the possible permutations of the roles. In this section we focus on such an m-player interaction, which allows us to relate strategies of the tournament and strategy combinations in the game $g$. This relationship is crucial as our object of analysis is the base game, and we want to investigate which of its equilibria are justified as limit equilibria of tournaments as $n$ increases.

The strategic interaction in which $m$ players play the base game $m$ ! times, simultaneously, and swapping roles in all their possible permutations can be directly represented by a symmetric normal form game $G$. Players have at least two ways of playing $G$. One possibility is to choose a probability distribution on $S$ (i.e., a mixed strategy) whose realization decides which action to take in all the matches played in each given role. Another, less general, possibility is to decide these $m$ actions independently through $m$ probability distributions (that we call "b-strategy"), one on $S_{1}$, one on $S_{2}$, and so on, each deciding the action to play in each possible role. We show below that these two ways of playing $G$ are equivalent in the sense that a player can generate the same scores by playing either mixed or $b$-strategies regardless of the strategy, and the type of strategy, that the other players are playing. This implies that a symmetric Nash equilibrium of the game $G$ is equivalent to a Nash equilibrium of $g .{ }^{10}$ Otherwise a player would have an incentive to deviate when playing in some given role. It also follows that every Nash equilibrium of $g$ can be transformed into a symmetric Nash equilibrium of $G$. As we observe in the next section, this allows us to extend some general properties of round-robin tournaments derived by AR13 to our tournaments.

Formally, take a group of $m$ players of the tournament. For each role $i$, let $Q_{-i}$ be the set of possible permutations of the $m-1$ roles different from $i$. Moreover, given a strategy combination $s^{o} \in S^{m-1}$ of $m-1$ opponents, let $s_{-i}^{o}(q)$ denote the resulting combination of actions in the match in which roles different from $i$ are assigned to those opponents according to $q \in Q_{-i}$. Then,

\footnotetext{
10 Here, we are identifying a symmetric Nash equilibrium with the corresponding strategy that is chosen by every player.
}

given the match $g=\left(M,\left(S_{i}\right)_{i \in M},\left(u_{i}\right)_{i \in M}\right)$, we can construct the $m$-player symmetric game $G:=(M, S, u)$, where

$$
u\left(s, s^{o}\right)=\sum_{i \in M} \sum_{q \in Q_{-i}} u_{i}\left(s_{i}, s_{-i}^{o}(q)\right)
$$

is the score to a player who plays strategy $s$ when her $m-1$ opponents play according to $s^{0}$.

The game $G$ summarizes the $m$ ! matches played by a group of $m$ players. Note that each player's mixed strategy set in $G$ coincides with the set of mixed strategies available in the tournament. However, our objective is to compare equilibria of the base game $g$ with equilibria of the tournament. To this end, we define a $b$-strategy $b=\left(b_{1}, \ldots, b_{m}\right)$ as a vector of $m$ probability distributions, one over each $S_{i}$. The set of all $b$-strategies is $B:=$ $\prod_{i \in M} \Delta\left(S_{i}\right)$. Given a $b$-strategy $b$, we define the corresponding product mixed strategy as the mixed strategy $\sigma_{b} \in \Delta(S)$ that satisfies $\sigma_{b}(s)=\prod_{i \in M} b\left(s_{i}\right)$ for every pure strategy $s \in S$. Note that the set of $b$-strategies $B$ is the set of mixed strategy combinations of the base game $g$. Thus, with slight abuse of notation, we will sometimes denote a typical strategy combination of $g$ by $b$ and a typical strategy of a player in the $m$-player interaction $G$ by $\sigma$.

We now prove that mixed and $b$-strategies are related through an analogue of Kuhn's theorem (Kuhn, 1953). ${ }^{11}$ As a consequence, every Nash equilibrium of $g$ can be turned into a symmetric Nash equilibrium of $G$, and every Nash equilibrium of $G$ can be described in terms of a $b$-strategy profile that is a Nash equilibrium of $g$. Let two (mixed or $b$-) strategies of a player be outcomeequivalent in the m-player interaction of the tournament if, for every (mixed or $b-$ ) strategy of the opponents, they induce the same expected score. We will sometimes refer to these strategies simply as equivalent strategies.

Proposition 1. In the m-player interaction of the tournament, for every mixed strategy $\sigma \in \Sigma$ there is an outcome-equivalent $b$-strategy $b \in B$, and vice versa. Moreover, given two equivalent strategies $b$ and $\sigma$, if $b=\left(b_{1}, \ldots, b_{m}\right)$ is a Nash equilibrium of $g$ then $(\sigma, \ldots, \sigma)$ is a Nash equilibrium of $G$, and vice versa.

Proof. Given the $m$-player match $g$ and $m$ players of the tournament, construct the extensive form game $E$ in which a fair move by Nature determines the roles in which those players are going to play $g$. That is, they play $g$ in all the possible permutations of the $m$ roles, each permutation having probability $1 / m ! .^{12}$ Let all the decision nodes in which a player plays in the same role belong to the same information set. Therefore, each player has $m$ information sets. The set of pure strategies of $E$ coincides with the set of pure strategies of $G$ and, at each pure strategy profile, the expected payoff profile in $E$ is equal to $1 / \mathrm{m}$ ! times the corresponding payoff profile in $G$. Moreover, the set of strategy combinations in $g$, i.e., the set of $b$-strategies, coincides with the set of behavioral strategies of $E$. Since $E$ has perfect recall, Kuhn's theorem directly implies that mixed and behavioral strategies of $E$ are realization equivalent. Hence, the first statement of the proposition readily follows. ${ }^{13}$

Now, let $b=\left(b_{1}, \ldots, b_{m}\right)$ be a Nash equilibrium of $g$ and consider the symmetric behavioral strategy combination $b^{*}=$

\footnotetext{
11 We cannot directly apply Kuhn's theorem given the lack of perfect recall. When a player chooses the action to take in the matches she plays in a given role, she does not know the outcome of the matches she plays in any other role.

12 For the case in which $m=2$, a direct proof of the proposition that does not rely on the auxiliary game $E$ can be found in the working paper version of this article. That can be extended to any $m>2$.

13 Note that we cannot construct tournaments directly on the game $E$ because it is the expected payoffs of $E$ that coincide with (a fraction of) the scores accumulated in the $m$-player interaction.
} 
$(b, \ldots, b)$ of the extensive form game $E$. The strategy combination $b^{*}$ is a Nash equilibrium of $E$, since no player has a profitable deviation at any of her information sets. It follows that all the symmetric mixed strategy combinations $(\sigma, \ldots, \sigma)$ such that $\sigma$ is equivalent to $b$ are Nash equilibria of $E$ and, therefore, of $G$. On the other hand, let $(\sigma, \ldots, \sigma)$ be a symmetric Nash equilibrium of $G$ and let the strategy $b$ be equivalent to $\sigma$. Then the symmetric behavioral strategy combination $(b, \ldots, b)$ is a Nash equilibrium of $E$. Indeed, in $E$, the expected payoff to a player at the information set corresponding to role $i$ and who chooses action $s_{i}$ given a symmetric strategy combination of the opponents is equal to $1 / \mathrm{m}$ times the score that player $i$ obtains in $g$ if she chooses action $s_{i}$ and her opponents choose the actions prescribed by that strategy combination. ${ }^{14}$ This implies that $b$ is a Nash equilibrium of $g$.

Remark 1. Of course, our symmetrization $G$ is only one of the possible symmetrizations that could be used. ${ }^{15}$ Arguably, it has the advantage of being intuitive and easily interpretable. Moreover, we can justify its choice by the previous proposition which allows to directly relate strategies and equilibria of the base game with strategies and equilibria of the tournament. In fact, the construction $G$ constitutes a useful link between our model and AR13's. In the next section, we prove that some results in AR13 extend to any $m$-player symmetric base game. Since strategies of $G$ and strategies of the tournament coincide, we can take $G$ as a base game of the tournament and, due to Proposition 1, express the results for the symmetric game $G$ in terms of equilibria of the original base game $g$.

\section{The tournament}

We can now study the relationship between equilibria of the game $g$ and equilibria of the tournament built on $g$. To this effect, the first step is to understand how strategies in $g$ and strategies in $T(g, n)$ are related. Recall that a player in the tournament is restricted to always play the same action in all the matches that she plays in the same role. Thus, like in the game $G$, a player in the tournament can choose to play a mixed strategy or a $b$ strategy (i.e. a mixed strategy with a product structure over $S$ ). But contrary to the game $G$, once we fix the behavior of the opponents, a mixed strategy in the tournament may not have a corresponding $b$-strategy that generates the same probability of winning the tournament even if it generates the same expected score. Furthermore, given a $b$-strategy $b$, there may be more than one mixed strategy that is equivalent to $b$ in the $m$-player interaction. In the tournament, these mixed strategies may also generate different winning probabilities. We illustrate these facts in the following example.

Example 1. Consider the double round-robin tournament with two players built on the following game $g$ :

$$
\begin{array}{c|c|c}
\multicolumn{1}{c}{} & \multicolumn{1}{c}{C} & D \\
\cline { 2 - 3 } A & 2,0 & 0,2 \\
\cline { 2 - 3 } B & 0,1 & 1,0
\end{array}
$$

14 In the expensive game $E$, each player plays in a given role with probability $1 / \mathrm{m}$, that is, the probability of each interaction played in that role $1 / \mathrm{m}$ ! multiplied by the number of these interactions $(m-1)$ !.

15 For example, a standard symmetrization of an $m \times n$ bimatrix game $(A, B)$ where, without loss of generality, $A$ and $B$ are the non-negative (and with no zero column) payoff matrices of the two players, is given by the $(m+n) \times(m+n)$ game $\left(C, C^{\top}\right.$ ) with $C=\left(\begin{array}{cc}0 & A \\ B^{\top} & 0\end{array}\right)$ (where $M^{\top}$ is the transpose of matrix $M$ ). Savani and von Stengel (2016, Proposition 2) prove that there exists a one-toone correspondence between equilibria of the bimatrix game and symmetric equilibria of such a symmetrization. However, this correspondence does not extend to all strategies (more precisely, to their strategy combinations and strategies, respectively).
The corresponding two-player symmetric game $G$ capturing the two-player interaction is (only payoffs to the first player are shown):

\begin{tabular}{c|c|c|c|c}
\multicolumn{1}{c}{} & \multicolumn{1}{c}{$A C$} & \multicolumn{1}{c}{$A D$} & \multicolumn{1}{c}{$B C$} & \multicolumn{1}{c}{$B D$} \\
\cline { 2 - 5 }$A C$ & 2 & 0 & 3 & 1 \\
\cline { 2 - 5 }$A D$ & 4 & 2 & 2 & 0 \\
\cline { 2 - 5 }$B C$ & 0 & 1 & 1 & 2 \\
\cline { 2 - 5 }$B D$ & 2 & 3 & 0 & 1
\end{tabular}

Since there are only two players in the tournament we can easily represent the $4 \times 4$ matrix with the probabilities of winning for each strategy profile (only the probability of winning of the first player is shown):

\begin{tabular}{c|c|c|c|c|}
\multicolumn{1}{c}{$A C$} & \multicolumn{1}{c}{$A D$} & \multicolumn{1}{c}{$B C$} & \multicolumn{1}{c}{$B D$} \\
\cline { 2 - 5 }$A C$ & $1 / 2$ & 0 & 1 & 0 \\
\cline { 2 - 5 }$A D$ & 1 & $1 / 2$ & 1 & 0 \\
\cline { 2 - 5 }$B C$ & 0 & 0 & $1 / 2$ & 1 \\
\cline { 2 - 5 }$B D$ & 1 & 1 & 0 & $1 / 2$
\end{tabular}

The match $g$ has a unique Nash equilibrium that generates the $b$-strategy $b=\left(\frac{1}{3} A+\frac{2}{3} B, \frac{1}{3} C+\frac{2}{3} D\right)$. We write such a $b$-strategy as the product mixed strategy $\sigma_{b}=\frac{1}{9} A C+\frac{2}{9} A D+\frac{2}{9} B C+\frac{4}{9} B D$. The non-product strategy $\sigma=\frac{1}{3} A C+\frac{2}{3} B D$ is also outcome-equivalent to $b$ in $G$. Proposition 1 implies that both $\left(\sigma_{b}, \sigma_{b}\right)$ and $(\sigma, \sigma)$ are Nash equilibria of $G$. But those two strategies are not equivalent in the tournament. For instance, $\sigma_{b}$ wins against $A C$ with probability $\frac{13}{18}$, while $\sigma$ wins against the same action with probability $\frac{5}{6}$.

Furthermore, this tournament does not have an equilibrium in $b$-strategies. This can be directly seen by checking that the unique equilibrium of the tournament is $\sigma^{*}=\frac{1}{3} A D+\frac{1}{3} B C+\frac{1}{3} B D$. But it is also illustrative to argue why in this example there cannot be an equilibrium in $b$-strategies. First, the tournament does not have any pure strategy equilibrium and, hence, an equilibrium in pure $b$-strategies does not exist. Moreover, there is no equilibrium in completely-mixed strategies because $A C$ is weakly dominated by $A D$ in the tournament. That is, there is no equilibrium in $b$-strategies where players completely mix between $A$ and $B$ at home and $C$ and $D$ away. For the same reason, the tournament does not have an equilibrium with support $\{A C, A D\}$, so there is no equilibrium in $b$-strategies where players play $A$ at home with probability one. Finally, no other equilibrium in $b$-strategies exists where players only randomize either at home or away because there is no equilibrium of the tournament with support $\{B C, B D\}$, $\{A C, B C\}$, or $\{A D, B D\}$.

Thus, we cannot define a tournament by restricting players to play the lower-dimensional set of $b$-strategies $B$ if we want to preserve existence of equilibria. ${ }^{16}$ This is one justification why, as outlined in Section 2, the strategy set must be the full dimensional set of mixed strategies. Proposition 1 gives us the proper tool to relate equilibria of the tournament $T(g, n)$ to equilibria of the base game $g$.

In particular, we relate the equilibria of a game to the limit equilibria of tournaments based on it in the following proposition, which establishes an analogous result to Proposition 1 in AR13. The limit of a sequence of equilibria of tournaments as $n$ goes to infinity is an equilibrium of the base game. First, we generalize AR13's proof to tournaments built on any m-player symmetric game, where every player meets each group of $m-1$ opponents to play the base game once. Second, we can consider $G$ to be such

\footnotetext{
16 Let $P\left(b^{\prime}, b\right)$ be the probability that a player playing $b^{\prime}$ wins the tournament when her opponents play according to $b$ (i.e., it is defined analogously to $\left.P\left(\sigma^{\prime}, \sigma\right)\right)$. The set $B$ is nonempty, compact, and convex. However, the function $P\left(b^{\prime}, b\right)$ is continuous in $b$ but not quasi-concave in $b^{\prime}$. The resulting nonconvexity of the best response correspondence is the reason why Nash equilibria in $b$-strategies may not exist.
} 
a base game and use Proposition 1 to translate equilibria of $G$ in terms of equilibria of $g .{ }^{17}$

Proposition 2. Let $\sigma$ be the limit point of a sequence of equilibria of $T(g, n)$ as $n$ goes to infinity. Then $\sigma$ is a (symmetric) Nash equilibrium of $G$ and the equivalent $b$-strategy $b=\left(b_{1}, \ldots, b_{m}\right)$ is a Nash equilibrium of $g$.

Proof. First, consider an $m$-player symmetric game $\tilde{G}$ and the $n$-player tournament $\tilde{R}(\tilde{G}, n)$ in which every player meets simultaneously each group of $m-1$ opponents once, and in every match the game $\tilde{G}$ is played. AR13 (Proposition 1 ) prove that, if $\tilde{G}$ is a two-player game, any strategy profile that is the limit point of some sequence of equilibria of $\tilde{R}(\tilde{G}, n)$ as $n$ goes to infinity is a symmetric equilibrium of $\tilde{G}$. We now generalize their proof to every $m>2$.

To this end, fix a player and let $\sigma^{0}$ be a symmetric strategy combination of the opponents, let $s^{0} \in S^{m-1}$ be a pure strategy combination of $m-1$ opponents and let $\sigma^{o}\left(s^{o}\right)$ be the probability that $\sigma^{o}$ attaches to it. For every pure strategy $s \in$ $S$, we denote with $u\left(s, \sigma^{0}\right)$ the random variable that receives value $u\left(s, s^{0}\right)$ with probability $\sigma^{o}\left(s^{0}\right)$. Then we have $\mathbb{E} u\left(s, \sigma^{0}\right)=$ $\sum_{s^{0} \in S^{m-1}} \sigma^{o}\left(s^{0}\right) u\left(s, s^{0}\right)$. Now, let $\sigma^{*}$ be the limit of a sequence $\left\{\sigma_{n}\right\}$ of equilibria of $\tilde{R}(\tilde{G}, n)$ as $n$ goes to infinity. Following AR13, we first show that every strategy in the support of $\sigma^{*}$ must induce the same expected score in $\tilde{G}$.

Assume to the contrary that $s$ and $s^{\prime}$ are pure strategies in the support of $\sigma^{*}$ such that $\mathbb{E} u\left(s^{\prime}, \sigma^{*}\right)>\mathbb{E} u\left(s, \sigma^{*}\right)$. For $n$ sufficiently large, we have $\mathbb{E} u\left(s^{\prime}, \sigma_{n}\right)>\mathbb{E} u\left(s, \sigma_{n}\right)+K$ for some $K>0$. Let $N_{0}=\left(\begin{array}{c}n-1 \\ m-1\end{array}\right)$ be the total number of matches that a player plays in the tournament, $N_{1}=\left(\begin{array}{c}n-2 \\ m-2\end{array}\right)$ be the number of matches in which she meets a given opponent, and $N_{2}=N_{0}-N_{1}$ be the number of matches in which she does not meet that opponent. When $m>2$, the scores that two players using $s$ and $s^{\prime}$ obtain when they meet each other are random variables because there are other players in the same match whose behavior is decided by the realization of a mixed strategy. Therefore, when players play according to strategy $\sigma_{n}$, the player who plays $s$ attains as high a score as the player who plays $s^{\prime}$ if

$\sum_{k=1}^{N_{2}} u\left(s, \sigma_{n}^{k}\right)+\sum_{j=1}^{N_{1}} u\left(s,\left(s^{\prime}, \sigma_{n}^{j}\right)\right) \geq \sum_{k=1}^{N_{2}} u\left(s^{\prime}, \sigma_{n}^{k}\right)+\sum_{j=1}^{N_{1}} u\left(s^{\prime},\left(s, \sigma_{n}^{j}\right)\right)$,

where $\sigma_{n}^{k}$ and $\sigma_{n}^{j}$ are copies of the $\tilde{R}(\tilde{G}, n)$ equilibrium strategy $\sigma_{n}$, and $u\left(s,\left(s^{\prime}, \sigma_{n}\right)\right)$ is the (random) score that a player who chooses $s$ gets in a match when one opponent chooses $s^{\prime}$ and all the others play according to $\sigma_{n}$ (similarly for $u\left(s^{\prime},\left(s, \sigma_{n}\right)\right)$ ). We can write the above condition as

$$
\begin{aligned}
& \sum_{k=1}^{N_{2}} u\left(s, \sigma_{n}^{k}\right)-\sum_{k=1}^{N_{2}} u\left(s^{\prime}, \sigma_{n}^{k}\right)-N_{2}\left[\mathbb{E} u\left(s, \sigma_{n}\right)-\mathbb{E} u\left(s^{\prime}, \sigma_{n}\right)\right] \geq \\
& \sum_{j=1}^{N_{1}} u\left(s^{\prime},\left(s, \sigma_{n}^{j}\right)\right)-\sum_{j=1}^{N_{1}} u\left(s,\left(s^{\prime}, \sigma_{n}^{j}\right)\right)+N_{2}\left[\mathbb{E} u\left(s^{\prime}, \sigma_{n}\right)-\mathbb{E} u\left(s, \sigma_{n}\right)\right] .
\end{aligned}
$$

Let $\overline{\bar{u}}=\max _{s^{o}} u\left(s,\left(s^{\prime}, s^{o}\right)\right)$ and $\bar{u}=\min _{s^{o}} u\left(s^{\prime},\left(s, s^{o}\right)\right)$ where $s^{o} \in S^{m-2}$. We can show that the probability of the event

$$
\begin{aligned}
& \sum_{k=1}^{N_{2}} u\left(s, \sigma_{n}^{k}\right)-\sum_{k=1}^{N_{2}} u\left(s^{\prime}, \sigma_{n}^{k}\right)-N_{2}\left[\mathbb{E} u\left(s, \sigma_{n}\right)-\mathbb{E} u\left(s^{\prime}, \sigma_{n}\right)\right] \\
& \quad \geq N_{1}(\bar{u}-\overline{\bar{u}})+N_{2} K
\end{aligned}
$$

\footnotetext{
17 Note that AR13's proof can be directly extended if $m=2$, as in this case
} $G$ is a symmetric two-player game. is of order $1 / n$, which implies that for $n$ sufficiently large the probability of (1) is of order $1 / n$.

For $n$ large enough, the right hand side of (2) is positive independently of the sign of $\bar{u}-\overline{\bar{u}}$ because $N_{2}$ is of order $n^{m-1}$ while $N_{1}$ is of order $n^{m-2}$. Thus, condition (2) is satisfied whenever the sum of random variables $\sum_{k=1}^{N_{2}}\left[u\left(s, \sigma_{n}^{k}\right)-u\left(s^{\prime}, \sigma_{n}^{k}\right)\right]$ exceeds its expectation $N_{2}\left[\mathbb{E} u\left(s, \sigma_{n}\right)-\mathbb{E} u\left(s^{\prime}, \sigma_{n}\right)\right]$ by $N_{1} L_{n}+N_{2} K>0$, where $L_{n}=\bar{u}-\overline{\bar{u}}$. Differently from the case $m=2$ (cf. AR13) when $m>2$ a player meets the same opponents in several matches. Since mixed strategies are executed only once, the random variables in the sum are not independent. ${ }^{18}$ These random variables have the same variance, that we denote $\mathbb{V}_{n}$, which converges to the variance of $u\left(s, \sigma^{*}\right)-u\left(s^{\prime}, \sigma^{*}\right)$. Recall that the covariance between two random variables is smaller than or equal to the highest of their variances. Finally, note that the (double of the) number of couples of dependent random variables is smaller than $N_{3}=N_{2}(m-1)\left(\begin{array}{c}n-3 \\ m-2\end{array}\right) .{ }^{19}$ It follows that the variance of $\sum_{k=1}^{N_{2}}\left[u\left(s, \sigma_{n}^{k}\right)-u\left(s^{\prime}, \sigma_{n}^{k}\right)\right]$ is smaller than $N_{2} \mathbb{V}_{n}+N_{3} \mathbb{V}_{n}$, being $N_{3} \mathbb{V}_{n}$ an upper bound of the covariances. Using Chebyshev's inequality, we obtain that the probability of the event (2) is smaller than $\frac{N_{2} \mathbb{V}_{n}+N_{3} \mathbb{V}_{n}}{\left(N_{1} L_{n}+N_{2} K\right)^{2}}$. Since $N_{3}$ is of order $n^{2 m-3}$ while $N_{2}{ }^{2}$ is of order $n^{2 m-2}$, such a fraction is of order $1 / n$.

Chernoff's inequality implies that with probability that rapidly approaches 1 there are at least $\frac{\sigma^{*}(s)}{2} n$ players who end up playing $s$. Hence, for sufficiently large $n$, the probability that a player who plays strategy $s$ wins the tournament is smaller than $c / n^{2}$ for some $c>0$. We conclude that all the strategies in the support of $\sigma^{*}$ must induce the same expected score in $\tilde{G}$ for $\sigma^{*}$ to be the limit of equilibria of tournaments $\tilde{R}(\tilde{G}, n)$ as $n$ goes to infinity.

Suppose now that the pure strategy $s^{\prime \prime}$ is not in the support of $\sigma^{*}$ and satisfies $\mathbb{E} u\left(s^{\prime \prime}, \sigma^{*}\right)>\mathbb{E} u\left(s, \sigma^{*}\right)$ for every $s$ in the support of $\sigma^{*}$. In this case, the argument above implies that the probability that a strategy $s$ in the support of $\sigma^{*}$ attains as high a score as $s^{\prime \prime}$ in $\tilde{R}(\tilde{G}, n)$ is smaller than $c_{s} / n$ for some $c_{s}>0$. Hence, letting $c_{*}$ be the maximum of these values, the probability that a player playing $s^{\prime \prime}$ wins the tournament when her $n-1$ opponents play according to $\sigma_{n}$ is greater than $\left(1-\frac{c_{*}}{n}\right)^{\# S}$, which is greater than $1 / n$ for $n$ sufficiently large (see also AR13).

We can therefore conclude that, given an $m$-player symmetric game $\tilde{G}$, every limit of subsequence of equilibria of $\tilde{R}(\tilde{G}, n)$ as $n$ goes to infinity is a symmetric equilibrium of $\tilde{G}$. Now, consider an $m$-player game $g$ and the corresponding symmetric game $G$ representing the $m$-player interaction in $T(g, n)$. It is easy to see that the tournament $T(g, n)$ based on $g$ coincides with the tournament $\tilde{R}(G, n)$ based on $G$. It follows that, if $\sigma^{*}$ is the limit point of a sequence of equilibria of $T(g, n)$ as $n$ goes to infinity, then $\sigma^{*}$ is a symmetric Nash equilibrium of $G$. By Proposition 1 , the $b$-strategy equivalent to $\sigma^{*}$ is a Nash equilibrium of $g$.

Proposition 2 tells us that the set of limit points of equilibria of tournaments as $n$ goes to infinity is a subset of the Nash equilibrium set of the base game. Proposition 3 shows that this is a strict subset of equilibria.

Proposition 3. Let $b=\left(b_{1}, \ldots, b_{m}\right)$ be a Nash equilibrium of $g$ and let $\Sigma(b)$ be the set of all mixed strategies that are equivalent

\footnotetext{
18 In fact, any two random variables $u\left(s, \sigma_{n}^{k}\right)-u\left(s^{\prime}, \sigma_{n}^{k}\right)$ and $u\left(s, \sigma_{n}^{k^{\prime}}\right)-u\left(s^{\prime}, \sigma_{n}^{k^{\prime}}\right)$ corresponding to two matches in which at least one opponent is the same are dependent, while any two random variables corresponding to two matches in which no opponent is the same are independent.

$19 N_{2}=\left(\begin{array}{c}n-2 \\ m-1\end{array}\right)$ is the total number of matches that a player plays with $n-2$ opponents. The number of these matches with the same given opponent is $\left(\begin{array}{c}n-3 \\ m-2\end{array}\right)$, and the number of possible opponents in the match is $m-1$. Note that in $N_{3}$ every couple of dependent random variables is counted twice.
} 
to $b$. The set $\Sigma(b)$ does not necessarily contain a limit point of a sequence of equilibria of $T(g, n)$ as $n$ goes to infinity.

Proof. The ultimatum game presented in Example 3 has a Nash equilibrium in which the first player proposes an unfair offer and the second player accepts both fair and unfair offers. Such a strategy is not a limit point of a sequence of equilibria of tournaments as the number of players increases. ${ }^{20}$

A Nash equilibrium $b$ of $g$ typically generates a set $\Sigma(b)$ of mixed strategies that are equivalent to $b$ in the $m$-player interaction. We now show that if a mixed strategy $\sigma \in \Sigma(b)$ is the limit point of a sequence of equilibria of tournaments as $n$ goes to infinity then it is not necessarily the case that all the strategies in $\Sigma(b)$ can be sustained in a similar way.

Example 2. Consider the double round-robin tournament based on the following game $g$ :

$$
\begin{array}{c|c|c|}
\multicolumn{1}{c}{C} & \multicolumn{1}{c}{D} \\
\cline { 2 - 3 } A & 0,0 & 1,-1 \\
\cline { 2 - 3 } B & 1,-1 & 0,0 \\
\cline { 2 - 3 }
\end{array}
$$

whose corresponding two-player symmetric game $G$ is:

\begin{tabular}{|c|c|c|c|c|}
\hline & $A C$ & $A D$ & $B C$ & $B D$ \\
\hline$A C$ & 0 & 1 & -1 & 0 \\
\hline$A D$ & -1 & 0 & 0 & 1 \\
\hline$B C$ & 1 & 0 & 0 & -1 \\
\hline$B D$ & 0 & -1 & 1 & 0 \\
\hline
\end{tabular}

The game $g$ has a unique Nash equilibrium, $b=\left(\frac{1}{2} A+\frac{1}{2} B, \frac{1}{2} C+\right.$ $\frac{1}{2} D$ ). Proposition 2 implies that at least one mixed strategy equivalent to $b$ is a limit point of a sequence of equilibria of $D(g, n)$ as $n$ goes to infinity. Indeed, we can see that the symmetries of the induced two-player interaction $G$ imply that $\sigma^{*}=\frac{1}{4} A C+\frac{1}{4} A D+$ $\frac{1}{4} B C+\frac{1}{4} B D$ is an equilibrium of the tournament for every $n$ and, consequently, also a limit point as $n$ goes to infinity.

But take the equivalent mixed strategy $\sigma=\frac{1}{2} A C+\frac{1}{2} B D$ and a sufficiently large number of players $n$. If the other $n-1$ players play according to $\sigma$, strategy $\frac{1}{2} A C+\frac{1}{2} B D$ accumulates a score equal to zero and wins the tournament with probability $1 / n$. In turn, strategy $A D$ 's accumulated score is equal to the number of players who end up playing $B D$ minus the number of players who end up playing $A C$. Such a score is strictly greater than 1 with probability close to $1 / 2$, while every other player's highest possible score is 1. Hence, a player who deviates to $A D$ also wins the tournament with probability close to $1 / 2$. An analogous reasoning is true for a deviation to strategy $B C$. It follows that $\sigma$ is never an equilibrium of $D(g, n)$ for large $n$, and it is not even a limit point of equilibria of tournaments as $n$ goes to infinity. In fact, since the probability of winning is continuous in the strategies of the opponents, the above argument implies that, for every $\tilde{\sigma} \in \Sigma$ and $\varepsilon$ small enough, strategy $(1-\varepsilon) \sigma+\varepsilon \tilde{\sigma}$ is not an equilibrium of the tournament with sufficiently large $n$.

\section{Tournament-stability}

Propositions 2 and 3 suggest a refinement criterion, which selects the Nash equilibria of a game that can be approximated by equilibria of tournaments built on it as the number of players increases. We call the equilibria that satisfy such a property tournament-stable equilibria. A tournament-stable equilibrium always exists. Indeed, every tournament has an equilibrium, so

\footnotetext{
20 Alternatively, one can consider the double round-robin tournament built on the "degenerate" game used to prove Proposition 2 in AR13. Note that such proposition cannot be directly extended to our framework, given our constraints on $G$.
}

for any sequence of tournaments $T(g, n)$ as $n$ goes to infinity we can construct an associated sequence of equilibria. Such a sequence is contained in the compact set $\Sigma$, hence it has a subsequence that converges. Thus, every game has a tournament-stable equilibrium.

Then, it seems natural to compare the solution concept of tournament-stable equilibrium with other concepts offered by the literature on equilibrium refinements, which have been introduced in order to capture some rationality principles. The next proposition shows that tournament-stability is satisfied by strict equilibria that, whenever they exist, satisfy all the nice properties one can hope for. A strict equilibrium is robust to various perturbations in the nature of the game and it is also robust to the current tournament specification.

Proposition 4. Let $b=\left(b_{1}, \ldots, b_{m}\right)$ be a strict Nash equilibrium of $g$, and let $s$ be its corresponding equivalent strategy. There exists an integer $\bar{n}$ such that $s$ is an equilibrium of the tournament $T(g, n)$ for every $n \geq \bar{n}$.

Proof. A Nash equilibrium is strict if every deviation implies a strict loss. If $b$ is a strict equilibrium in $g$ then its equivalent strategy $s$ is a strict equilibrium in $G$. With slight abuse of terminology, let $s$ denote also the strategy combination of the opponents in which everybody chooses $s$, and let $\left(s^{\prime}, s\right)$ denote the strategy combination in which one opponent chooses $s^{\prime}$ and all the others choose $s$. Denote with $k=\min _{s^{\prime} \neq s} u(s, s)-u\left(s^{\prime}, s\right)>0$ the minimum loss that a player incurs in any $m$-player interaction if she deviates from $s$, and with $\bar{k}=\max _{s^{\prime} \neq s} u(s, s)-u\left(s,\left(s^{\prime}, s\right)\right)$ the maximum loss (or the minimum gain) of the opponents that she can induce by deviating. Since a player in the tournament participates in $\left(\begin{array}{c}n-1 \\ m-1\end{array}\right) m$-player interactions (summarized by the symmetric game $G$ ) and every opponent meets her $\left(\begin{array}{c}n-2 \\ m-2\end{array}\right)$ times, a player does not have incentive to deviate from $s$ if $\left(\begin{array}{c}n-1 \\ m-1\end{array}\right) k \geq$ $\left(\begin{array}{c}n-2 \\ m-2\end{array}\right) \bar{k}$. That is, if $n \geq \frac{\bar{k}(m-1)+k}{k}$. Letting $\bar{n}$ be the smallest integer greater than or equal to this fraction, the result follows.

Not surprisingly, the set of strict equilibria is a strict subset of the set of tournament-stable equilibria. Nonetheless, tournamentstable equilibria may exclude strategy combinations that are strategically stable. Indeed, in the following example tournamentstability eliminates an equilibrium that is strictly perfect, hence, also perfect and a Kohlberg and Mertens (1986)'s stable set. Moreover, such an equilibrium is also proper and a Mertens (1989)'s stable set. That is, tournament-stability may not select an equilibrium that satisfies the most prominent concepts proposed by the equilibrium refinement literature.

Example 3 (The Ultimatum Game). Consider the double roundrobin tournament whose match $g$ coincides with the "ultimatum game". The player playing at home can offer a fair $(F)$ or unfair $(U)$ proposal about how to split 10 dollars, and the player playing away can either accept $(A)$ or reject $(R)$ it. The utility for a player to have $x$ dollars is given by $x$.

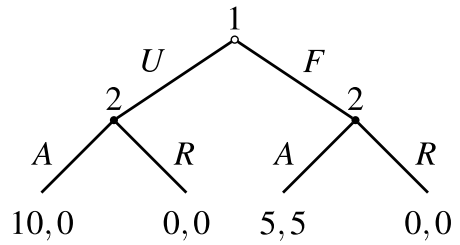

Hence, the match $g$ is the normal form game:

\begin{tabular}{c|c|c|c|c}
\multicolumn{1}{c}{} & \multicolumn{1}{c}{$A A$} & \multicolumn{1}{c}{$A R$} & \multicolumn{1}{c}{$R A$} & \multicolumn{1}{c}{$R R$} \\
\cline { 2 - 5 }$U$ & 10,0 & 10,0 & 0,0 & 0,0 \\
\cline { 2 - 5 }$F$ & 5,5 & 0,0 & 5,5 & 0,0 \\
\cline { 2 - 5 } & &
\end{tabular}

Strategy FRA - make a fair proposal and accept a proposal if and only if it is fair - is an equilibrium of the tournament for every 
$n$, and the equivalent $b$-strategy $(F, R A)$ is an undominated Nash equilibrium of $g$. On the other hand, the $b$-strategy $(U, A A)-$ make an unfair proposal and accept both fair and unfair proposals - is a strictly perfect equilibrium of $g$ and, therefore, a stable set as defined by Kohlberg and Mertens (1986). It also satisfies the stronger stability definition proposed by Mertens (1989). The equivalent strategy $U A A$, however, is not an equilibrium of the tournament for any $n$, and neither is any close-by strategy. Indeed, in the matches in which a player plays away, she has the incentive to deviate and reject the unfair offer to inflict a loss of 10 to her opponents. Hence, if everybody conforms to $U A A$, a player can increase her probability of winning from $1 / n$ to 1 by deviating to URA. Therefore strategy UAA cannot not even be approached by a limit point of equilibria of tournaments as the number of players increases. It follows that a stable set does not necessarily contain an equilibrium of the tournament $T(g, n)$ for any $n$, and not even a limit point of a sequence of equilibria as $n$ goes to infinity.

Note that, in this version of the ultimatum game, rejecting an unfair proposal is costless because it does not have any effect on the total score of the player doing it. It also inflicts a loss to the other player. We now modify the ultimatum game used as the match of the tournament so that a player can offer a "somewhat unfair" proposal that is costly to reject because it entails giving up the small split that is offered. Suppose that the player playing at home can additionally make an intermediate offer $(M)$ that gives some amount $z$ to the player playing away, with $0<z<5$ :

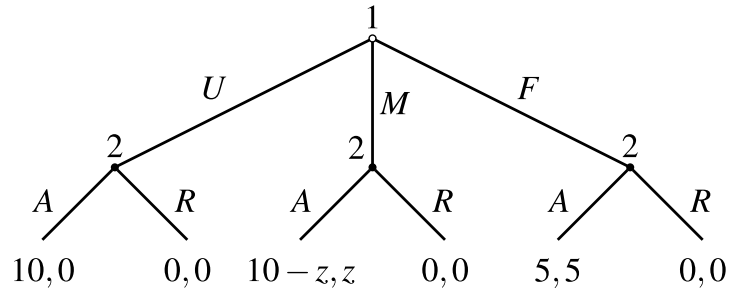

As before, offering the fair proposal and accepting a proposal only if it is fair is an equilibrium of the tournament for every $n$ and every $z$. Likewise, the tournament has no equilibrium in which players accept the unfair proposal $U$. Now, however, accepting the intermediate offer $M$ can be part of an equilibrium strategy of the tournament, but only if the number of players is sufficiently large. Indeed, for each $z$, strategy $M A A$ is an equilibrium of the tournament $D(g(z), n)$ if and only if $n \geq n_{z}=\left\lceil\frac{10}{z}\right\rceil$. Note that $\lim _{z \rightarrow 0} n_{z}=\infty$, so arbitrarily unfair proposals can be sustained in equilibrium provided the size of the population is large enough.

Even if, in this example, tournament-stability seems to be extremely restrictive, it may also allow for equilibria that are not strategically stable. In the following example we show that every dominated equilibrium of the base game is an equilibrium of the tournament for large enough $n$.

Example 4 (The Entry Game). Consider the classical "entry game", in which Firm 1 decides whether or not to enter the market, and Firm 2 decides how to compete, either aggressively (Fight) or not (Accommodate). Specifically, let the extensive form representation of the game be:

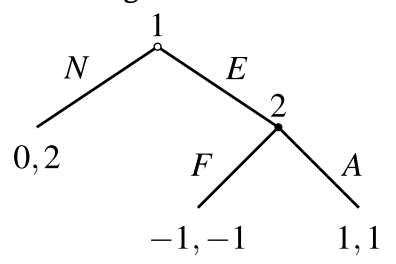

whose corresponding normal form representation of the match $g$ is:

\begin{tabular}{c|c|c|}
\multicolumn{1}{c}{} & \multicolumn{1}{c}{$F$} & \multicolumn{1}{c}{$A$} \\
\cline { 2 - 3 }$N$ & 0,2 & 0,2 \\
\cline { 2 - 3 }$E$ & $-1,-1$ & 1,1 \\
\cline { 2 - 3 }
\end{tabular}

The match $g$ has an undominated Nash equilibrium $(E, A)$ which is subgame perfect in the extensive form representation. The equivalent strategy $E A$ is an equilibrium of the tournament $D(g, n)$ for every $n \geq 3$. But $g$ also has a continuum of dominated Nash equilibria,

$E^{*} \equiv\left\{(N, \alpha F+(1-\alpha) A): \frac{1}{2} \leq \alpha \leq 1\right\}$.

Of course, equilibria in $E^{*}$ are not subgame perfect equilibria because Firm 2 is making a non-credible threat about its intention to fight if Firm 1 enters the market. However, in the tournament $D(g, n)$, strategy $N F$ - not entering the market under the threat of a fight - is an equilibrium when $n \geq 4$.

Let us now analyze strategies in the continuum $E^{*}$ and how they can be sustained as equilibria of the tournament. Under the mixed strategy $\alpha N F+(1-\alpha) N A$, where $\frac{1}{2} \leq \alpha \leq 1$, the only potential profitable deviation to consider is playing $E$ instead of $N$ when in the role of the first firm. Thus, for a given $\alpha$, consider a player who plays $E$ when in the role of Firm 1 while all the other players play strategy $\alpha N F+(1-\alpha) N A$ (clearly, in this case, strategies $E F$ and $E A$ attain the same score and the same probability of winning). Let $x$ be the number of players that play $N F$ in equilibrium. The player who plays $E$ accumulates a score of $n-1-2 x$ at home and a score of $2(n-1)$ away for a total of $3 n-3-2 x$. In turn, players who play $N A$ accumulate $2(n-2)+1$ and players who play $N F$ accumulate $2(n-2)-1$, so a player who ends up playing $N F$ does not win the tournament if there is at least one player who plays NA. Thus, playing $E$ when in the role of the first firm is a profitable deviation if and only if

$\mathbb{P}\left(x=\frac{n}{2}\right) \frac{1}{n-x}+\mathbb{P}\left(x<\frac{n}{2}\right)>\frac{1}{n}$,

where

$\mathbb{P}\left(x=\frac{n}{2}\right)=\left(\begin{array}{c}n-1 \\ n / 2\end{array}\right) \alpha^{n / 2}(1-\alpha)^{n-1-n / 2}$

is positive only if $n$ is an even number and, letting $m=\left\lceil\frac{n}{2}\right\rceil$,

$\mathbb{P}\left(x<\frac{n}{2}\right)=\sum_{k=0, \ldots, m-1}\left(\begin{array}{c}n-1 \\ k\end{array}\right) \alpha^{k}(1-\alpha)^{n-1-k}$.

When $\alpha=\frac{1}{2}, E$ is always a profitable deviation, so strategy $\frac{1}{2} N F+\frac{1}{2} N A$ is never an equilibrium of the tournament for any $n$. For a fixed $n>4$, the left hand side of (3) is decreasing in $\alpha$ and equals the right hand side at some value $\alpha_{n}^{*} \in\left(\frac{1}{2}, 1\right)$. It follows that, for every $n>4,\left\{\alpha N F+(1-\alpha) N A: \alpha_{n}^{*} \leq \alpha \leq 1\right\}$ is a continuum of equilibria of the tournament $D(g, n)$. In particular, $\alpha_{n}^{*}$ is decreasing in $n$ and approaches $\frac{1}{2}$ as $n$ grows to infinity. We conclude that all the strategies in the continuum, including $\frac{1}{2} N F+\frac{1}{2} N A$, are limit points of equilibria of tournaments as $n$ goes to infinity. That is, even if each dominated equilibrium is a "non-credible threat" in the match, such threats are rendered plausible in the tournament provided the number of interactions is sufficiently high.

Thus, apart from selecting a strict equilibrium when it exists, there is no other systematic relationship between tournamentstability and traditional equilibrium refinements. As mentioned by AR13, the refinement criterion provided by tournaments may be more appropriate to an evolutionary interpretation of equilibrium. To this end and in their same vein, in the next section we 
examine the intuition that tournaments encourage risky behavior, using a very natural setting in which tournaments prove very useful to refine the Nash equilibrium set.

\section{Taking risks}

In the same spirit as AR13, Section 5, we now explore the intuition that tournaments encourage risk-taking behavior. Our construction allows us to study such an intuition using a different avenue. We analyze a two-player game with a continuum of equilibria that induce a continuum of different risk levels associated to the choice of one of the players, and we show that the unique tournament-stable equilibrium is the riskiest one, thus suggesting a very useful refinement criterion. In contrast, AR13 consider a $2 \times 2$ symmetric game that has a unique symmetric equilibrium in which players randomize between a risky and a safe action, and they examine how this equilibrium is approached in tournaments as the number of players increase; that is, how often the risky action is chosen in the equilibrium of the tournament with respect to the equilibrium of the base game. Note that in this case the probabilities with which an individual chooses the risky and the safe actions are those that balance the equilibrium payoffs of the opponents, and in this sense they depend on the incentives of the others. Arguably, the current approach has the advantage to make individual incentives more explicit, as the equilibrium associated with the riskiest behavior is precisely the only one that survives. Moreover, these incentives are unambiguously driven by the tournament structure, i.e., by the fact that the prize is allocated to the player who accumulates the highest score. To highlight this, we briefly consider different tournament incentives and assume that players play to minimize the probability of attaining the lowest score. Under this revised tournament structure, the only equilibrium that survives is the one associated with the safest behavior.

Consider a two-player match where the player playing at home can either take an outside option or play a constant sum game against the other player. Scores have been chosen so that the outside option gives a score equal to the value of the constant sum game for the home player. In this sense, the outside option is the safe action $(S)$ while choosing to play the constant sum game is the risky action $(R)$.

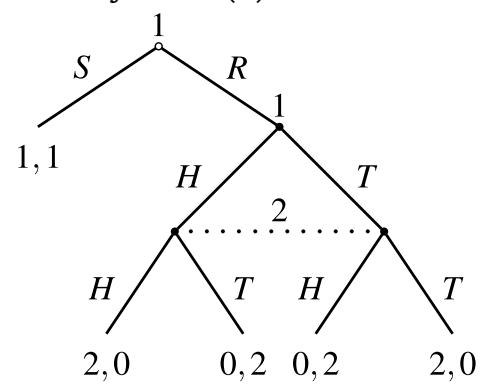

The match $g$ is therefore:

$$
\begin{array}{r|c|c}
\multicolumn{1}{c}{} & \multicolumn{1}{c}{H} & \multicolumn{1}{c}{T} \\
\cline { 2 - 3 } S & 1,1 & 1,1 \\
\cline { 2 - 3 } R H & 2,0 & 0,2 \\
\cline { 2 - 3 } R T & 0,2 & 2,0 \\
\cline { 2 - 3 } & &
\end{array}
$$

The set of Nash equilibria of $g$ consists of the continuum of strategy profiles

$$
\left\{\left((1-2 x) S+x R H+x R T, \frac{1}{2} H+\frac{1}{2} T\right): 0 \leq x \leq \frac{1}{2}\right\} \text {. }
$$

In every equilibrium point, player 1 randomizes between the safe outside option $S$ and entering the subgame playing its equilibrium strategy $\frac{1}{2} H+\frac{1}{2} T$. Of course, player 2 responds with its corresponding equilibrium strategy $\frac{1}{2} H+\frac{1}{2} T$. Every strategy profile in the one-dimensional set of equilibria induces the same expected scores and, moreover, the set of equilibria can be ordered in terms of second order stochastic dominance, from the one giving the least risky returns to the first player $(x=0)$ to the one giving her the riskiest returns $(x=1 / 2)$. We can show that, in the associated tournament, only this last equilibrium survives as the number of players increases.

Indeed, Proposition 2 implies that, for large enough $n$, every player plays close to $\frac{1}{2} H+\frac{1}{2} T$ when playing away. If all players play $S$ at home then they all attain the same score and win with probability $1 / n$. But when a player deviates to, e.g., $R H$ at home she obtains the highest score whenever more players end up playing $H$ than $T$ when playing away. And that event happens with probability close to $1 / 2$. A similar argument goes through for every equilibrium of $g$ in which the first player plays the safe action with positive probability.

It is revealing to briefly consider a tournament structure built on $g$ that generates more "conservative" incentives, and to examine how that affects the players' risk-taking behavior in equilibrium. To this effect, suppose that players' objective is not anymore to maximize the probability of being the highest scorer in the tournament but to minimize the probability of being the lowest scorer. If several players obtain the lowest score, the loser is selected at random. One can prove that the analogous version of Proposition 2 also holds with this tournament structure. That is, any limit point of equilibria of tournaments as $n$ goes to infinity is a Nash equilibrium of $g$. This implies that, for large enough $n$ and in equilibrium, every player plays a strategy close to $\frac{1}{2} H+\frac{1}{2} T$ in the matches away. Suppose that players play a strategy close to $\frac{1}{2} R H+\frac{1}{2} R T$ when playing at home. Under such a strategy profile, any given action is the lowest scorer with probability close to $1 / 4$. For example, playing $R H$ at home and $H$ away accumulates the lowest score if more players end up playing $T$ than $H$ away and also more players end up playing $R H$ than $R T$ at home.

Now, consider a player who deviates to the safe action $S$ at home. Define a configuration $\mu$ to be a possible realization in the tournament of the mixed strategy profile indicated above and, for any action at home and away $A$, let $\mu(A)$ be the number of players who choose it. The score accumulated by the deviating player is

$(n-1)+ \begin{cases}2 \mu(R H) & \text { if she ends up playing } T \\ 2 \mu(R T) & \text { if she ends up playing } H .\end{cases}$

In turn, the score accumulated by each of the other possible actions is

$$
\begin{aligned}
(R H, H): & 2(\mu(H)-1)+2 \mu(R T)+1=2 \mu(H)+2 \mu(R T)-1 \\
(R H, T): & 2 \mu(H)+2(\mu(R H)-1)+1=2 \mu(H)+2 \mu(R H)-1 \\
(R T, H): & 2 \mu(T)+2(\mu(R T)-1)+1=2 \mu(T)+2 \mu(R T)-1 \\
(R T, T): & 2(\mu(T)-1)+2 \mu(R H)+1=2 \mu(T)+2 \mu(R H)-1 .
\end{aligned}
$$

Note that the probability that at least one player plays each of these actions rapidly approaches 1 as $n$ increases. Suppose that the configuration $\mu$ is such that $\mu(T) \leq \mu(H)$. Then, the lowest score among the non-deviating players is obtained by those who end up playing either $(R T, H)$ or $(R T, T)$. If additionally $\mu(R T) \leq$ $\mu(R H)$ then the lowest score in the tournament is less than or equal to $(n-1)+2 \mu(R T)-1$, which is strictly smaller than the score accumulated by the deviating player computed in Eq. (4) regardless of the action that she plays away. An analogous reasoning goes through if $\mu(R T) \geq \mu(R H)$ or $\mu(T) \geq \mu(H)$. Thus, we conclude that deviating to the safe action $S$ at home is a profitable deviation in the tournament whenever players play $\frac{1}{2} R H+\frac{1}{2} R T$ at home and $\frac{1}{2} H+\frac{1}{2} T$ away. A similar argument works for every strategy in the continuum of Nash equilibria of the match $g$ such that $x>0$. Therefore, in the unique Nash equilibrium of the match that is a limit point of equilibria of tournaments as $n$ goes to infinity, players play the safe action at home. 


\section{7. (Double) round-robin tournaments}

It is evident from the above applications that our construction extends the scope of AR13's analysis from two-player symmetric games to a very broad range of games. Moreover, it allows us to examine also non-symmetric equilibria of symmetric games. We conclude studying some two-player symmetric games, for which we can compare the results obtained with our double roundrobin tournaments and those obtained with AR13's round robins. From such a study it emerges that the extension of AR13's results to our setting is not immediate but requires careful study.

Consider first the coordination game $g$ :

$$
\begin{array}{c|c|c}
\multicolumn{1}{c}{} & \multicolumn{1}{c}{A} & \multicolumn{1}{c}{B} \\
\cline { 2 - 3 } A & 1,1 & 0,0 \\
\cline { 2 - 3 } B & 0,0 & 1,1 \\
\cline { 2 - 3 }
\end{array}
$$

AR13 (Proposition 4) prove that equilibria of $g$ and equilibria of the round-robin tournament built on $g$ coincide for any number of players. Hence, the strategy profile in which every player randomizes equally between playing $A$ in every match and playing $B$ in every match is an equilibrium of the tournament for every $n$. Now, consider the double round-robin tournament based on $g$, whose two-player interaction is summarized by the game

\begin{tabular}{c|c|c|c|c}
\multicolumn{1}{c}{} & \multicolumn{1}{c}{$A A$} & \multicolumn{1}{c}{$A B$} & \multicolumn{1}{c}{$B A$} & \multicolumn{1}{c}{$B B$} \\
\cline { 2 - 5 }$A A$ & 2 & 1 & 1 & 0 \\
\cline { 2 - 5 }$A B$ & 1 & 0 & 2 & 1 \\
\cline { 2 - 5 }$B A$ & 1 & 2 & 0 & 1 \\
\cline { 2 - 5 }$B B$ & 0 & 1 & 1 & 2 \\
\cline { 2 - 5 } & & &
\end{tabular}

and take the strategy profile in which, in the same vein as before, every player randomizes equally between playing $A$ in every match and playing $B$ in every match, i.e. plays strategy $\sigma=$ $\frac{1}{2} A A+\frac{1}{2} B B$. Then, assume that the total number of players is odd and consider a player who contemplates the configuration in which half of her opponents end up playing $A A$ and the other half end up playing $B B$. Under this configuration, strategy $A B$ attains the same score that $A A$ and $B B$ would do if the player chose them; but it decreases the score that $A A$ and $B B$ obtain by 1 . Since in this case $A B$ wins with probability 1 and the probability of such a realization is larger than $1 / n$ (because such a realization is the mode of the binomial $B(n-1, p)$, whose associated probability is larger than $1 /(n-1)>1 / n)$, it follows that $A B$ is a profitable deviation and, hence, $\sigma$ is not an equilibrium of the tournament. ${ }^{21}$

Now, let us just relabel the actions of one of the players in $g$ so that we obtain the strategically equivalent symmetric game $g^{\prime}$ :

$$
\begin{array}{c|c|c}
\multicolumn{1}{c}{} & \multicolumn{1}{c}{A} & \multicolumn{1}{c}{B} \\
\cline { 2 - 3 } A & 0,0 & 1,1 \\
\cline { 2 - 3 } B & 1,1 & 0,0
\end{array}
$$

First, note that building a double round-robin tournament on $g^{\prime}$ allows us to study also the non-symmetric equilibria $(A, B)$ and $(B, A)$ which are excluded in AR13's analysis. ${ }^{22}$ In particular, it directly follows from the result in Proposition 4 that their equivalent strategies $A B$ and $B A$ are symmetric Nash equilibria of $D\left(g^{\prime}, n\right)$ for every $n$ sufficiently large. Then, note that $g^{\prime}$ has a unique symmetric Nash equilibrium, $b=\left(\frac{1}{2} A+\frac{1}{2} B, \frac{1}{2} A+\frac{1}{2} B\right)$, which is the unique equilibrium of the corresponding roundrobin tournament $R\left(g^{\prime}, n\right)$ for every $n$ (see AR13, p.40). But, in this case, the strategy in which every player randomizes equally between playing $A$ in every match and playing $B$ in every match is also an equilibrium of the double round robin based on $g^{\prime}$. In fact, we can show that, for every $n$ large enough, when players

\footnotetext{
21 In turn, one can show that $\sigma$ is an equilibrium of the tournament when the number of players is even.

22 see also footnote 7 .
}

play according to $\sigma=\frac{1}{2} A A+\frac{1}{2} B B$ there is no profitable deviation neither to action $A B$ nor to action $B A$, and this is enough to conclude that $\sigma$ is an equilibrium of $D\left(g^{\prime}, n\right)$ because $b$ is an equilibrium of $R\left(g^{\prime}, n\right)$.

Indeed, given the two-player interaction $G^{\prime}$

\begin{tabular}{c|c|c|c|c}
\multicolumn{1}{c}{} & \multicolumn{1}{c}{$A A$} & \multicolumn{1}{c}{$A B$} & \multicolumn{1}{c}{$B A$} & \multicolumn{1}{c}{$B B$} \\
\cline { 2 - 5 }$A A$ & 0 & 1 & 1 & 2 \\
\cline { 2 - 5 }$A B$ & 1 & 2 & 0 & 1 \\
\cline { 2 - 5 }$B A$ & 1 & 0 & 2 & 1 \\
\cline { 2 - 5 }$B B$ & 2 & 1 & 1 & 0 \\
\cline { 2 - 5 } & & & &
\end{tabular}

it is easy to see that the symmetric game obtained from $G^{\prime}$ after eliminating actions $A B$ and $B A$ is an affine transformation of $g^{\prime}$. Therefore, no player has an incentive to deviate to either $A A$ or $B B$ in $D\left(g^{\prime}, n\right)$. Moreover, actions $A B$ and $B A$ are equivalent given $\sigma$, so that it is enough to examine the incentives to deviate to $A B$. Action $A B$ attains the maximum score only when every opponent chooses $A A$ or when every opponent chooses $B B$, winning in both cases with probability 1 . That is, when $x$ opponents play $A A$ and $n-x-1$ opponents play $B B$, the player who plays $A B$ obtains a score equal to $n-1$, while players playing $A A$ and $B B$ obtain, respectively, $2 n-2 x-1$ and $2 x+1$, so $A B$ never wins the tournament. It follows that, when players play according to $\sigma$, $A B$ wins the tournament with probability $(1 / 2)^{n-1}+(1 / 2)^{n-1}$. Consider now the winning probability of, e.g., $A A$. It wins with probability $1 / n$ when every opponent plays $A A$, with probability 1 when every opponent plays $B B$, and with probability $1 /(x+1)$ (resp. $1 / n$ ) when $x$ opponents play $A A, n-x-1$ opponents play $B B$ and $x<\frac{n}{2}-1$ (resp. $x=\frac{n}{2}-1$ ). For $n$ large enough, the probability that $A A$ wins the tournament when players play according to $\sigma$ is therefore greater than

$$
\begin{aligned}
\left(\frac{1}{2}\right)^{n-1}+\frac{1}{2} \mathbb{P}(x=1) & =\left(\frac{1}{2}\right)^{n-1}+\frac{1}{2} \frac{1}{2}\left(\frac{1}{2}\right)^{n-2}(n-1) \\
& =\left(\frac{1}{2}\right)^{n-1}+\frac{n-1}{2}\left(\frac{1}{2}\right)^{n-1},
\end{aligned}
$$

which is greater than the probability of winning of $A B$. We conclude that, contrary to the case in which the base game was $g$, now $\sigma$ is supported as equilibrium of every double round-robin tournament in which the number of players is sufficiently large. ${ }^{23}$

23 The results of AR13 also remain valid with our construction for the unique symmetric equilibrium of the "game of chicken" $g$ :

\begin{tabular}{c|c|c|} 
& \multicolumn{1}{c}{$A$} & \multicolumn{1}{c}{$B$} \\
\cline { 2 - 3 }$A$ & 0,0 & $-1,1$ \\
\cline { 2 - 3 }$B$ & $1,-1$ & $-10,-10$ \\
\cline { 2 - 3 } & &
\end{tabular}

Strategy $b=\left(\frac{9}{10} A+\frac{1}{10} B, \frac{9}{10} A+\frac{1}{10} B\right)$ is the limit point of a sequence of equilibria of round-robin tournaments as $n$ goes to infinity and, analogously, the equivalent strategy $\sigma=\left(\frac{9}{10} A A+\frac{1}{10} B B\right)$ is the limit point of a sequence of equilibria of double round-robin tournaments. To see this we take, for every $n$, the unique symmetric equilibrium $\alpha_{n} A+\left(1-\alpha_{n}\right) B$ of $R(g, n)$ and construct strategy $\sigma_{n}=\alpha_{n} A A+\left(1-\alpha_{n}\right) B B$. Then we check whether there are profitable deviations in $D(g, n)$ from $\sigma_{n}$ to either $A B$ or $B A$. As before, both of these tournament strategies win with probability 1 under the configurations in which either all the opponents end up playing $A A$ or all the opponents end up playing $B B$. Namely, they both win with probability $\alpha_{n}^{n-1}+\left(1-\alpha_{n}\right)^{n-1}$. On the other hand, action $B B$ wins with probability 1 when every other player plays $A A$ and with probability $1 / 2$ when only one other player plays $B B$ (it also wins under other configurations but we can neglect them here). That is, it wins with probability greater than $\alpha_{n}^{n-1}+\frac{n-1}{2} \alpha_{n}^{n-2}\left(1-\alpha_{n}\right)$. Since $\alpha_{n}>1 / 2$ (see AR13, Proposition 6), we can conclude that $\sigma_{n}$ is an equilibrium also for $D(g, n)$ and, therefore, $\sigma$ is justified as a limit equilibrium also with double round-robin tournaments. Note again that, under our construction, the strict non-symmetric Nash equilibria of $g$ play a role too as they are sustained as equilibria of tournaments with a sufficiently large number of players.

The same reasoning and the same results can be easily extended to general $2 \times 2$ anti-coordination games. 


\section{Acknowledgments}

We thank Bernhard von Stengel for his useful suggestions. We also thank the editor and two anonymous referees for comments that improved the paper. Carlos acknowledges financial support from the Australian Research Council's Discovery Project DP190102629. The usual disclaimer applies.

\section{References}

Arad, A., Rubinstein, A., 2013. Strategic tournaments. Am. Econ. J. Microecon. 5 31-54.

Dixit, A.K., 1987. Strategic behavior in contests. Amer. Econ. Rev. 77, 891-898. Green, J.R., Stokey, N.L., 1983. A comparison of tournaments and contracts. J. Political Econ. 91, 349-364.

Groh, C., Moldovanu, B., Sela, A., Sunde, U., 2012. Optimal seedings in elimination tournaments. Econ. Theory 49, 59-80.
Kohlberg, E., Mertens, J.-F., 1986. On the strategic stability of equilibria. Econometrica 54, 1003-1038.

Konrad, K.A., 2004. Bidding in hierarchies. Eur. Econ. Rev. 48, 1301-1308.

Konrad, K.A., 2009. Strategy and Dynamics in Contests. Oxford University Press, New York.

Kuhn, H., 1953. Extensive games and the problem of information. In: Kuhn, H., Tucker, A. (Eds.), Contributions to the Theory of Games, vol. 2. pp. 193-216.

Laffond, G., Laslier, J.-F., Le Breton, M., 2000. K-player additive extension of twoplayer games with an application to the borda electoral competition game. Theory Decis 48, 129-137.

Maynard Smith, J., Parker, G.A., 1976. The logic of asymmetric contests. Anim. Behav. 24, 159-175

Mertens, J.-F., 1989. Stable equilibria-a reformulation, part I: Definition and basic properties. Math. Oper. Res. 14 (4), 575-625.

Rosen, S., 1986. Prizes and incentives in elimination tournaments. Amer. Econ. Rev. 76, 701-715.

Savani, R., von Stengel, B., 2016. Unit vector games. Int. J. Econ. Theory 12 , 7-27.

van Damme, E., 1991. Stability and Perfection of Nash Equilibria. Springer-Verlag, Berlin. 